Conclusion The majority of physicians indicate that they can adequately estimate a patient's limited life expectancy and that they tend to discuss patients' wishes if they have a poor prognosis. Information transfer concerning patients' wishes for treatment and care can be improved.

\section{OP64 EXAMINING PATIENT-REPORTED BARRIERS TO TALKING ABOUT ADVANCE CARE PLANNING (ACP) WITH FAMILY PHYSICIANS: A MULTI-SITE SURVEY}

A Tan*, C Bernard, M Slaven, M Howard, D Elston, D Heyland. Cumming School of Medicine, University of Calgary, Calgary, Canada

\subsection{6/spcare-2019-ACPICONGRESSABS.64}

Background Advance care planning (ACP) can improve satisfaction with end-of-life care among patients and families and reduce unwanted treatments. Primary care is an ideal setting in which to facilitate ACP. This study analyzed the reasons why patients find it difficult to discuss ACP with their family physicians.

Methods A self-completed, validated questionnaire about four ACP engagement behaviours and barriers was administered to patients aged 50 and older in 20 family practices in Canada. The questionnaire included an open-ended question about what makes it difficult to talk about ACP with the family physician. Four authors analysed the open-ended comments using thematic content analysis.

Results 810 patients (mean age $=66,55.6 \%$ female) participated. Of the $53 \%(n=428)$ of patients who had talked to someone about end-of-life medical treatments, only $18 \%$ $(n=75)$ had talked with their family physician. Patients identified the following barriers to ACP conversations: 1) They feel too young, healthy and well; 2) They abdicate responsibility to their physician; 3) They worry about a negative impact of ACP on the physician relationship; 4) Inadequate time during appointments; 5) They feel ACP is emotionally difficult to discuss with their physician.

Conclusions Our findings suggest that patients need help preparing for ACP conversations, both to change the perception that ACP conversations only occur at the end-of-life and to normalize these discussions between patients and physicians. There is an opportunity for family physicians, who have longstanding relationships and frequent visits with patients, to have ACP conversations.

\section{OP65 FRAMING ADVANCE CARE PLANNING IN PARKINSON'S DISEASE: PATIENT AND CARE PARTNER PERSPECTIVES}

${ }^{1} \mathrm{H}$ Lum*, 'S Jordan, ${ }^{1} \mathrm{~A}$ Brungardt, ${ }^{2} \mathrm{R}$ Ayele, ${ }^{3} \mathrm{M}$ Katz, ${ }^{4} \mathrm{~J}$ Miyasaki, ${ }^{3} \mathrm{~A}$ Hall, ${ }^{2} \mathrm{~J}$ Jones, ${ }^{5} \mathrm{~B}$ Kluger. 'University of Colorado and VA GRECC, Aurora, USA; ${ }^{2}$ University of Colorado College of Nursing, Aurora, USA; ${ }^{3}$ University of California San Francisco, San Francisco, USA; ${ }^{4}$ University of Alberta, Edmonton, Canada; ${ }^{5}$ university of Colorado School of Medicine, Aurora, USA

\subsection{6/spcare-2019-ACPICONGRESSABS.65}

Background Advance care planning (ACP) is a new core quality measure in caring for individuals with Parkinson's disease (PD) and there are no best practice standards for how to incorporate the ACP process into PD care. This study describes patient and care partner perspectives on ACP to inform a patient and care partner-centered framework for clinical care.
Methods Qualitative descriptive study of 30 patients and 30 care partners affected by PD within a multi-site, randomized clinical trial of neuropalliative care compared to standard care. Participants were individually interviewed about perspectives on ACP, including prior and current experiences, barriers to ACP, and suggestions for integration into care. Interviews were analyzed using theme analysis to identify key themes.

Results Four themes illustrate how ACP is perceived and integrated into clinical care: 1) variation in personal definitions of ACP in the context of PD; 2) barriers to engaging in ACP with $\mathrm{PD}$; 3) role of care partners as active participants in ACP; and 4) influence of a palliative care approach on ACP. Taken together, the themes support clinician initiation of ACP discussions and interdisciplinary approaches to help patients and care partners overcome barriers to ACP.

Conclusions ACP in PD may be influenced by patient and care partner perceptions and misperceptions, symptoms of PD (e.g. apathy, cognitive dysfunction, disease severity), and models of clinical care. Optimal engagement of PD patients and care partners in ACP should proactively address misperceptions of ACP and utilize clinic teams and workflow routines to incorporate ACP into regular care.

\section{OP66 HOW DO PATIENTS WITH LIFE LIMITING DISEASES EXPERIENCE PATIENT-PHYSICIAN COMMUNICATION ABOUT LIFE EXPECTANCY? - AN INTERVIEW STUDY}

${ }^{1} \mathrm{C}$ Owusuaa, ${ }^{1} \mathrm{~L}$ van Lent, ${ }^{1} \mathrm{C}$ van der Rijt, ${ }^{2} \mathrm{~A}$ van der Heide. 'Erasmus MC Cancer Institute, Rotterdam, Netherlands; ' Department of Public Health, Erasmus MC, Rotterdam, The Netherlands

\subsection{6/spcare-2019-ACPICONGRESSABS.66}

Background The Dutch Framework for Palliative Care advises physicians to timely start advance care planning (ACP) in patients with life limiting diseases. Such communication requires disclosure and discussion of patients' limited life expectancy. We explored patients' experiences with such discussions.

Methods Medical specialists from three different hospitals included patients with incurable cancer or late-stage chronic obstructive pulmonary disease (COPD), with whom they had spoken about their limited life expectancy. All 14 patients (7 cancer and 7 COPD) had a semi-structured interview with one researcher about their experiences on those conversations. The interviews were audiotaped, transcribed, coded, and analysed by two researchers.

Results All patients were aware of their limited life expectancy. They were often shocked when their physician had indicated prognosis rather concrete. However, such indications also enabled them to reflect certain treatments and led to conversations about ACP sometimes. Most patients agreed that the physician should initiate conversations about life expectancy, but that the patient should have control of the continuation of that topic. Some patients with COPD who disagreed with this also believed that the pulmonologist lacked the ability to indicate their life expectancy. Factors that facilitated agreeable conversations for the patients were: clear explanations and messages about the disease, prognosis and treatment options, feeling of being heard, sufficient time, and adequate preparation by the physician, an open attitude, and sensitive non-verbal communication. 
Conclusion Overall, patients appreciate honest, personalized and attentive communication on a limited life expectancy enabling reflections and communications about ACP.

\section{OP67 PATIENT PERSPECTIVES ON INFORMATION PROVISION AND ADVANCE CARE PLANNING REGARDING IMPLANTABLE CARDIOVERTER DEFIBRILLATOR DEACTIVATION AT THE END OF LIFE}

R Stoevelaar*, A Brinkman-Stoppelenburg, R Bhagwandien, R Van Bruchem-Visser, D Theuns, A van der Heide, J Rietjens. Erasmus MC, Rotterdam, Netherlands

\subsection{6/spcare-2019-ACPICONGRESSABS.67}

Background Implantable Cardioverter Defibrillator (ICD) shocks can negatively influence the last phase of life. Advance care planning, timely and frequently discussing ICD deactivation with the patient, can help patients to make a wellinformed decision about future ICD deactivation. Perspectives of patients on ICD deactivation are however largely unknown. Methods Focus groups with ICD patients were conducted using a predefined topic list and were audiotaped and transcribed. Transcripts were analyzed using the constant comparative method.

Results Forty-one patients participated in a total of five focus groups. Average age was 64 years and $56 \%$ of patients were male. Many patients expressed a need for more information about ICD deactivation. Although most patients agreed that it would be appropriate to deactivate the ICD on a certain point in the disease trajectory, they had difficulties deciding on the right moment to do so, indicating the need to frequently re-assess their preferences. Possible circumstances in which patients would consider deactivating their ICD in order to avoid shocks were: a terminal stage of their disease, and a diminished quality of life. Arguments against deactivation were also mentioned, and mainly concerned not wanting to give up on life, both for themselves and for their family.

Conclusion(s) There is room for improvement in informing patients about ICD deactivation in the last phase of life. We found that patients' perspectives towards ICD deactivation were highly personal and sometimes ambivalent. This emphasizes the importance of early and recurring discussions on this topic.

\section{OP68 A CLUSTER-RANDOMIZED TRIAL OF A NURSE-LED ADVANCE CARE PLANNING SESSION IN PATIENTS WITH COPD AND THEIR LOVED ONES}

${ }^{1} \mathrm{C}$ Houben*, ${ }^{1} \mathrm{M}$ Spruit, ${ }^{2} \mathrm{H}$ Luyten, ${ }^{3} \mathrm{H}$ Pennings, ${ }^{4} \mathrm{~V}$ van den Boogaart, ${ }^{5} \mathrm{~J}$ Creemers, ${ }^{6} \mathrm{G}$ Wesseling, ${ }^{6} \mathrm{E}$ Wouters, ${ }^{1} \mathrm{D}$ Janssen. ${ }^{1} \mathrm{C}$ iro, Horn, Netherlands; ${ }^{2}$ University of Twente, Enschede, Netherlands; ${ }^{3}$ St Laurentius Hospital, Roermond, Netherlands; ${ }^{4}$ VieCuri Medical Centre, Venlo, Netherlands; ${ }^{5}$ Catharina Hospital Eindhoven, Eindhoven, Netherlands; ${ }^{6}$ Maastricht UMC+, Maastricht, Netherlands

\subsection{6/spcare-2019-ACPICONGRESSABS.68}

Background Advance Care Planning (ACP) is uncommon in patients with COPD.

Aims To assess whether a nurse-led ACP-intervention can improve quality of patient-physician end-of-life care communication in patients with COPD. Furthermore, the influence of an ACP-intervention on symptoms of anxiety and depression in patients and loved ones was studied.
Methods A multicenter cluster randomized-controlled trial (RCT) in patients with advanced COPD was performed. The intervention group received an 1.5-hours structured nurse-led ACP-session. Outcomes were: quality of patient-physician endof-life care communication, prevalence of ACP-discussions six months after baseline, and symptoms of anxiety and depression in patients and loved ones.

Results 165 patients were enrolled (89 intervention: mean age 65.7 (9.2) years; 49.4\% male; 76 control: mean age 69.5 (9.0) years; $57.9 \%$ male). The improvement of quality of patient-physician end-of-life care communication was significantly higher in the intervention group compared to the control group (<.001). The ACP-intervention was significantly associated with the occurrence of an ACP-discussion with physicians within 6 months $(\mathrm{p}=.003)$. At follow-up, symptoms of anxiety were significantly lower in loved ones in the intervention group compared to the control group $(p=.02)$. Symptoms of anxiety in patients, and symptoms of depression in both patients and loved ones were comparable at follow-up ( $\mathrm{p}>.05)$.

Conclusion One nurse-led ACP-intervention session improves patient-physician end-of-life care communication without causing psychosocial distress in both patients and loved ones.

The Advance Care Planning study is supported by Lung Foundation Netherlands.

\section{OP69 EFFECTIVENESS OF ADVANCE CARE PLANNING IN IMPROVING END OF LIFE CARE FOR PATIENTS WITH ADVANCED HEART FAILURE}

C Malhotra*, D Sim, F Jaufeerally, N Nadkarni, H Meibo, E Finkelstein. Duke-NUS Medical School, Singapore, Singapore

\subsection{6/spcare-2019-ACPICONGRESSABS.69}

Background Our primary aim was to assess, among advanced heart failure patients, effectiveness of Advance care planning (ACP) in ensuring end of life (EOL) care consistent with patient wishes. Secondary aims were to assess its impact on patients' decisional conflict, discussion of care preferences with surrogates, illness understanding, anxiety, depression and quality of life.

Methods We conducted a randomized controlled trial of ACP (based on Respecting Choices Model) versus usual care in Singapore. 282 patients hospitalized with heart failure and NYHA III and IV symptoms were randomized to ACP (93) or control (189) arm. They answered up to 6 followup surveys conducted every 4 months. Primary outcome was assessed in the deceased sample $(89 ; 23$ in ACP, 66 in control arm). Both intention-to-treat and per-protocol analyses were done.

Results 63\% of ACP arm received intervention. Deceased patients in ACP arm were no more likely to have their wishes followed for EOL treatments (35\% in ACP vs $44 \%$ in control; $\mathrm{p}=0.47$ ) but were more likely to have their wishes followed for cardiopulmonary resuscitation $(83 \%$ in ACP vs $62 \%$ in control, $p=0.12$ ) though the difference was not statistically significant. At first follow-up, ACP patients had lower decisional conflict $(\beta=-10.8, p<0.01)$ and were more likely to discuss preferences with their surrogate decision maker $(\beta=1.3, p=0.04)$. Both arms did not differ on other patient outcomes. Per-protocol analyses showed similar results. 